\title{
INFLUÊNCIA DA POSIÇÃO DOS INSTRUMENTOS DE MEDIDA NA DETERMINAÇÃO DO MÓDULO DE ELASTICIDADE DA MADEIRA NA COMPRESSÃo PARALELA ÀS FIBRAS $\left(\mathrm{E}_{\mathrm{C} 0}\right)^{1}$
}

Fabiane Salles Ferro ${ }^{2}$, Felipe Hideyoshi Icimoto ${ }^{2}$, Diego Henrique Almeida ${ }^{3}$, André Luís Christoforo ${ }^{4}$ e Francisco Antonio Rocco Lahr ${ }^{5}$

\begin{abstract}
RESUMO - O módulo de elasticidade na compressão paralela às fibras $\left(\mathrm{E}_{\mathrm{c} 0}\right)$ é um dos parâmetros de referência para estimar o desempenho da madeira. O Anexo B da Norma Brasileira ABNT NBR 7190:1997 estabelece, nos ensaios para determinação do $\mathrm{E}_{\mathrm{c} 0}$, que seja tomada a medida de deformações em pelo menos duas faces opostas dos corpos de prova. O objetivo deste trabalho foi verificar a influência dessas condições de ensaio nos valores de $\mathrm{E}_{\mathrm{c} 0}$. Tal propriedade foi determinada a partir das deformações de duas faces opostas dos corpos de prova e, em seguida, a partir das deformações obtidas nas faces complementares. Foram utilizadas espécies de madeira de forma a abranger todas as classes de resistência assumidas pela citada norma. Os resultados indicam significativa variação de $\mathrm{E}_{\mathrm{c} 0}$ determinado nos ensaios referidos, evidenciando que tal situação deve ser considerada para futura revisão dos métodos de ensaio para determinação de propriedades da madeira para aplicação estrutural.
\end{abstract}

Palavras-chave: Compressão paralela às fibras; Módulo de elasticidade; Madeira.

\section{INFLUENCE OF THE POSITION OF THE MEASURING INSTRUMENTS IN THE DETERMINATION OF THE ELASTICITY MODULUS OF WOOD IN COMPRESSION PARALLEL TO GRAIN ( $\left.E_{C O}\right)$}

\begin{abstract}
The modulus of elasticity in compression parallel to the grain $\left(E_{c 0}\right)$ is a reference parameter to estimate the performance of the timber. Annex B of the Brazilian Code ABNT NBR 7190:1997 establishes, for determining the $E_{c 0}$, to measure deformations at least on two opposite sides of the specimens. The aim of this study was to assess the influence of these test conditions in $E_{c 0}$ values. This property was determined based on two opposite sides deformations of the specimens, and then, from the deformations obtained in complementary sides. Wood species were used to cover all strength classes assumed by this standard. Results showed a significant variation of $E_{c 0}$ determined in mentioned tests, demonstrating that such a situation should be considered for future revision of the test methods for the determination of wood properties for structural application.
\end{abstract}

Keywords: Compression parallel to grain; Elasticity modulus; Wood.

\footnotetext{
${ }^{1}$ Recebido em 28.05.2013 aceito para publicação em 25.03.2015.

${ }^{2}$ Universidade de São Paulo, USP, Programa de Pós-graduação em Ciências e Engenharia de Materiais, SP, Brasil. E-mail: <fsferro@usp.br>e<icimoto@usp.br>.

${ }^{3}$ Universidade de São Paulo, USP, Programa de Pós-graduação em Engenharia Civil, São Carlos, SP, Brasil. E-mail: <almeidadh@usp.br>.

${ }^{4}$ Universidade Federal de São Carlos, UFSCar, Departamento de Engenharia Civil, São Carlos, SP, Brasil. E-mail: <alchristoforo@yahoo.com.br>.

${ }^{5}$ Universidade de São Paulo, USP, Departamento de Engenharia de Estruturas, São Carlos, SP, Brasil. E-mail: <frocco@sc.usp.br>.
} 


\section{INTRODUÇ̃̃O}

A madeira é material heterogêneo que possui diferentes tipos de tecidos, adaptados para desempenharem funções específicas. As variações nas composições químicas, físicas e anatômicas da madeira são significativas e ocorrem entre espécies, dentro de uma espécie e, até mesmo, no interior de uma árvore. A heterogeneidade da madeira está relacionada com diversos fatores, como aspectos climáticos, solo, local de crescimento e de ordem genética, assim como aspectos anatômicos e de crescimento (ROCHA, 1994; TRUGILHO et al., 1996; CALIL JUNIOR et al., 2003).

$\mathrm{Na}$ madeira existem diferenças entre a região do cerne e alburno, madeira de início e o fim de estação de crescimento, lenhos juvenil e adulto e em escala microscópica, entre células individuais (TRUGILHO et al., 1996).

Muitos estudos abordam a influência da variabilidade da madeira em suas propriedades mecânicas.

Ballarin e Palma (2003) verificaram que as propriedades de resistência e rigidez da madeira adulta em relação à juvenil de Pinus taeda são maiores em cerca de $54 \%$ e $47 \%$, respectivamente.

Stanzl-Tschegg et al. (2010) verificaram que a tolerância à fratura da madeira de reação em relação à madeira normal das espécies Taxus baccata e Picea abies é menor, pois existem tensões residuais resultantes das condições de desenvolvimento da árvore.

Também nesse sentido, encontra-se o estudo de Gryc e Vavrèík (2009), que avaliaram a variabilidade da espécie Picea abies na resistência à compressão paralela às fibras para madeiras normais e de reação. Concluíram que a madeira de reação apresentou valores de resistência ligeiramente superiores em relação à madeira normal. A largura dos anéis de crescimento também foi significativa nessa propriedade, de forma que os valores de resistência à compressão paralela diminuíram com o aumento da largura dos anéis.

Outras considerações similares sobre a influência da variabilidade da madeira em suas propriedades mecânicas podem ser encontradas nos trabalhos de Kretschmann (2008) e Vidaurre (2011).

A variabilidade da madeira reflete-se essencialmente em suas propriedades físicas e mecânicas (LAHR, 1990). Conhecê-la é fundamental para definir adequadamente as aplicações a que serão destinadas a dimensionar, com segurança, as partes componentes de uma estrutura (ARAÚJO, 2007).

No Brasil, as condições de ensaios para determinação das propriedades físicas e mecânicas da madeira para projetos de estruturas constam na Norma NBR 7190 (ABNT, 1997). Essa norma utiliza como principal referência para as propriedades mecânicas o ensaio de compressão paralela às fibras, a partir do qual se determinam a resistência e rigidez da madeira.

A compressão paralela às fibras é importante parâmetro na concepção de elementos estruturais. A madeira, quando submetida a esses esforços, comporta-se de maneira dúctil, não possui ruptura catastrófica e também é resistente em níveis de solicitação entre os mais elevados que o material consegue desempenhar (SILVA et al., 2012).

O Anexo B da referida norma, denominado "Determinação das propriedades das madeiras para projeto de estruturas", assume que, para determinação do módulo de elasticidade na compressão paralela às fibras, devem ser realizadas medidas de deformações em pelo menos duas faces opostas dos corpos de prova, pois, dessa forma, obtém-se uma média representativa da deformação da madeira. A norma não define a orientação da seção transversal do corpo de prova para realização do ensaio, ou seja, não leva em consideração a anisotropia e a heterogeneidade. Nessas circunstâncias, os módulos de elasticidade determinados podem não representar a real condição da madeira, uma vez que as faces escolhidas podem não abranger totalmente a variabilidade natural da madeira presente em todo o volume do corpo de prova.

Assim, o objetivo deste trabalho foi verificar a influência das condições de ensaios requeridas pela Norma NBR 7190 (ABNT, 1997) nos valores do módulo de elasticidade da madeira, na compressão paralela às fibras.

\section{MATERIAL E MÉTODOS}

O trabalho foi desenvolvido no Laboratório de Madeiras e de Estruturas de Madeira (LaMEM), do Departamento de Engenharia de Estruturas (SET) da Escola de Engenharia de São Carlos (EESC) e da Universidade de São Paulo (USP).

Para a realização deste estudo, foram utilizadas nove espécies de madeira, de modo que abrangessem 
todas as classes de resistência assumidas pela Norma. Classe C20: cambará-rosa (Erisma sp.); Classe C30: eucalipto grandis (Eucalyptus grandis), Pinus elliottii (Pinus elliottii) e cedro (Cedrella sp.); Classe C40: cupiúba (Goupia glabra) e eucalipto citriodora (Corymbia citriodora); Classe C50: ipê (Tabebuia sp.); Classe C60: jatobá (Hymenaea stilbocarpa); e angicorosa (Anadenanthera falcata).

Foram utilizados oito corpos de prova de compressão paralela às fibras de cada espécie analisada. Assim como estabelecido pela Norma em questão, os corpos de prova foram extraídos de peças diferentes de um lote considerado homogêneo.

Todas as peças de madeira das quais se retiraram os corpos de prova apresentavam aproximadamente $12 \%$ de umidade. Os ensaios foram procedidos de acordo com ABNT NBR 7190:1997. Os corpos de prova foram confeccionados com seção transversal nominal quadrada de $5 \mathrm{~cm}$ de lado e comprimento de $15 \mathrm{~cm}$. As medidas dos lados dos corpos de prova foram realizadas com um paquímetro com sensibilidade de $0,001 \mathrm{~mm}$.

Para determinação do módulo de elasticidade, foram realizadas medidas de deformações em duas faces opostas do corpo de prova e, em seguida, nas outras duas faces complementares. As faces que apresentaram menor média do módulo de elasticidade foram denominadas A e as faces que apresentaram a maior média do módulo de elasticidade, B. Esse foi o único critério utilizado para definir as faces dos corpos de prova de compressão paralela às fibras.

Os ensaios foram realizados em uma máquina universal de ensaios AMSLER, com capacidade de carga de $250 \mathrm{kN}$, e foram procedidos até $50 \%$ da força estimada de ruptura, para garantir que o limite de proporcionalidade (regime elástico) de cada amostra não fosse ultrapassado. As deformações foram medidas com o auxílio de relógios comparadores com sensibilidade de $0,001 \mathrm{~mm}$, fixados por meio de cantoneiras metálicas. Assim, cada corpo de prova foi ensaiado duas vezes, pois a Norma NBR 7190:1997 sugere que, para determinação do MOE na compressão paralela às fibras, sejam realizadas medidas de deformação em apenas duas faces opostas do corpo de prova.

O módulo de elasticidade foi determinado pela inclinação da reta secante à curva tensão x deformação, utilizando-se a equação 1 .

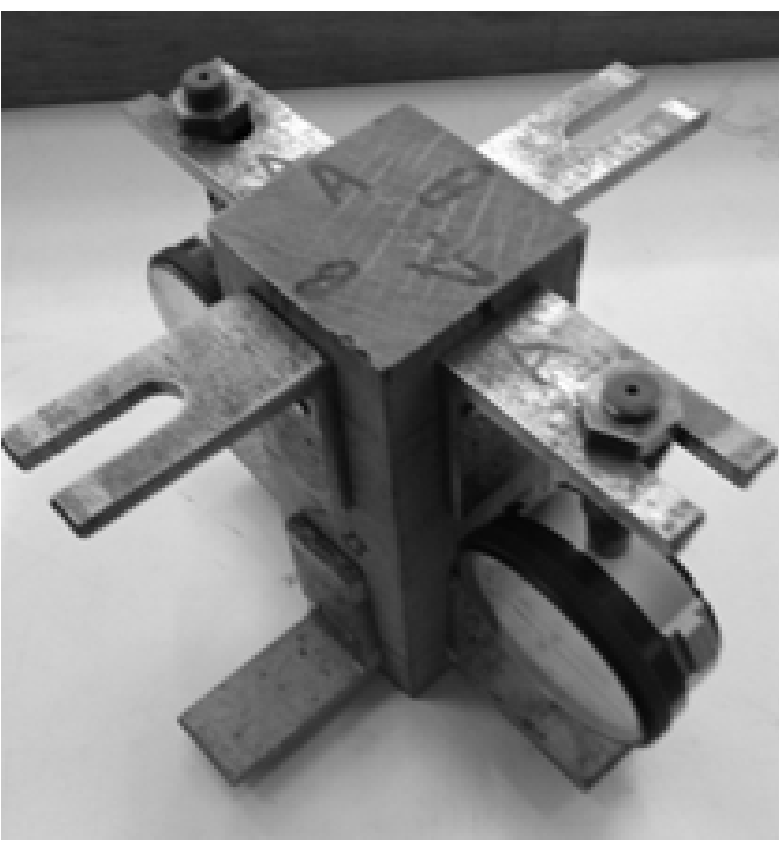

Figura 1 - Faces "A" e "B" do corpo de prova após o ensaio de compressão paralela às fibras.

Figure 1-Sides" $A$ " and " $B$ " in thespecimen after the compression parallel to grain test.

$$
E_{c 0}=\frac{\sigma_{50 \%}-\sigma_{10 \%}}{\xi_{50 \%}-\xi_{10 \%}}
$$

$\mathrm{Na}$ equação $1, \sigma_{10 \%}$ é a tensão correspondente a $10 \%$ da resistência à compressão paralela às fibras, $\sigma_{50 \%}$ é a tensão correspondente a $50 \%$ da resistência à compressão paralela às fibras, $\xi_{10 \%}$ é a deformação específica medida no corpo de prova, correspondente à tensão de $\sigma_{10 \%}$; e $\xi_{50 \%}$ é a deformação específica medida no corpo de prova correspondente à tensão de $\sigma_{50 \%}$.

Para cada corpo de prova analisado no ensaio de compressão paralela às fibras, foram obtidos dois valores do módulo de elasticidade $\left(\mathrm{E}_{\mathrm{c} 0}\right)$, correspondentes às faces $\mathrm{A}$ e $\mathrm{B}$.

Para verificação da influência das posições (A e B) dos relógios comparadores no cálculo dos módulos de elasticidade na compressão paralela às fibras $\left(\mathrm{E}_{\mathrm{c} 0}\right)$, em cada corpo de prova ensaiado dividiu-se o menor valor do módulo de elasticidade $\left(\mathrm{E}_{\mathrm{c} 0 \_} \mathrm{A}\right)$ pelo maior valor $\left(\mathrm{E}_{\mathrm{c} 0-} \mathrm{B}\right)$, possibilitando realizar uma análise dos dados independentes da espécie e da respectiva classe de resistência, utilizando-se teste de hipótese, com

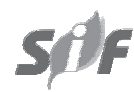

Revista Árvore, Viçosa-MG, v.39, n.4, p.743-749, 2015 
o auxílio do software Minitab $(14$ como forma de avaliar a influência das posições dos relógios no cálculo da rigidez.

Constatada a não equivalência estatística entre os módulos de elasticidade, utilizou-se o método dos mínimos quadrados (equação 2), possibilitando determinar o coeficiente ótimo baseado na ideia de menor resíduo (CHRISTOFORO et al., 2012).

$$
f(\gamma)=\frac{1}{2} \cdot \sum(\text { Razão }-\gamma \cdot \text { Unidade })^{2}
$$

$\mathrm{Na}$ equação $2, f(\gamma)$ é a função do método dos mínimos quadrados; $\gamma$ é o coeficiente ótimo; Razão é o vetor constituído de valores inferiores a 1 (razão entre os menores valores dos módulos de elasticidade pelos maiores valores encontrados por corpo de prova); e Unidade é o vetor de componentes iguais a 1 (valor esperado da razão entre os módulos de elasticidade).

\section{RESULTADOS}

Na Tabela 1 são apresentados os resultados dos valores médios dos módulos de elasticidade de cada espécie de madeira obtidos com a fixação dos relógios comparadores nas posições A (menor valor - $\mathrm{E}_{\mathrm{c} 0 \_} \mathrm{A}$ ) e B (maior valor - $\mathrm{E}_{\mathrm{c} 0 \_} \mathrm{B}$ ), sendo Min e Máx os menores e os maiores valores encontrados por resposta, respectivamente.

De acordo com a Tabela 1, a maior variação dos valores médios dos módulos de elasticidade na compressão paralela às fibras foi obtida pela espécie Cupiúba, com 14.595 MPa para as faces A e 17.779 MPa para as faces B (aproximadamente 22\%). Já a menor variação do $\mathrm{E}_{\mathrm{co}}$ foi obtida pela espécie ipê, com valores médios de 17.608 e $17.935 \mathrm{MPa}$, respectivamente para as faces A e B (cerca de $2 \%$ ).

Os resultados obtidos da razão entre os módulos de elasticidade $\mathrm{E}_{\mathrm{c} 0 \_} \mathrm{A}$ e $\mathrm{E}_{\mathrm{c} 0 \_} \mathrm{B}$ são: média das razões $\left(\mathrm{x}_{\mathrm{m}}\right)$ igual a 0,9077 , desvio-padrão $(\mathrm{Sd})$ igual a 0,0776 e coeficiente de variação $(\mathrm{Cv})$ igual a $8,55 \%$. Os valores mínimo e máximo da razão entre as diferenças de módulos de elasticidade foram iguais a 0,6298 e 1 , respectivamente.

A Figura 2 ilustra o resultado do teste de normalidade de Anderson-Darling sobre a razão entre os módulos de elasticidade. O nível de significância (á) do teste é de $5 \%$, consistindo em normalidade dos dados para a hipótese nula $\left(\mathrm{H}_{0}\right)$ e não normalidade dos dados para a hipótese alternativa $\left(\mathrm{H}_{1}\right)$. Dessa forma, $\mathrm{P}$-valor do teste superior em nível de significância implica aceitar $\mathrm{H}_{0}$, rejeitando-a em caso contrário.

Pelo fato de o P-valor encontrado na Figura 2 ser inferior a $0,05(5 \%)$, constata-se não ser normal a distribuição da razão entre os módulos de elasticidade, ressaltando ser a normalidade dos dados o requisito exigido para o emprego do teste de hipótese. Nessas condições, foi utilizada a Transformada de Johnson (Figura 3), cuja transformação é apresentada pela equação 3 , sendo $\mathrm{X}$ a razão entre os módulos de elasticidade e Y, o valor encontrado na transformação.

$$
Y=-1.80093+0.981792 \cdot \log \left(\frac{X-0.44795}{100784-X}\right)
$$

A Figura 4 ilustra o resultado do teste de normalidade de Anderson-Darling dos dados transformados. Pelo fato de P-valor encontrado ser superior a 5\%, constata-se

Tabela 1 - Valores médios dos módulos de elasticidade das nove espécies estudadas.

\begin{tabular}{|c|c|c|c|c|c|c|}
\hline \multirow{2}{*}{ Espécie } & \multicolumn{3}{|c|}{ Relógios comparadores nas posições A } & \multicolumn{3}{|c|}{ Relógios comparadores nas posições B } \\
\hline & Média $\mathrm{E}_{\mathrm{c} 0}(\mathrm{MPa})$ & Min & $\operatorname{Max}$ & Média $\mathrm{E}_{\mathrm{c} 0}(\mathrm{MPa})$ & Min & $\operatorname{Max}$ \\
\hline Angico-rosa & 21988 & 15514 & 28070 & 23902 & 15943 & 31444 \\
\hline Cambará-rosa & 13234 & 8500 & 18690 & 14501 & 10546 & 18795 \\
\hline Cedro & 10837 & 9915 & 13152 & 11899 & 10859 & 13152 \\
\hline Cupiúba & 14595 & 10991 & 18805 & 17779 & 12779 & 20888 \\
\hline Eucalipto citriodora & 15683 & 11592 & 19352 & 17878 & 15256 & 24705 \\
\hline Eucalipto grandis & 11239 & 9730 & 12676 & 11892 & 10466 & 13681 \\
\hline Ipê & 17608 & 16122 & 19529 & 17935 & 16676 & 19813 \\
\hline Jatobá & 20264 & 17454 & 24695 & 22045 & 18750 & 28565 \\
\hline Pinus elliottii & 11560 & 7913 & 16560 & 13490 & 8697 & 19837 \\
\hline
\end{tabular}

Table 1 - Mean values of elasticity modulus for the nine species studied.

Revista Árvore, Viçosa-MG, v.39, n.4, p.743-749, 2015 
ser normal a distribuição das razões entre os módulos de elasticidade, validando o emprego do teste de hipótese.

A unidade, valor esperado da razão entre os módulos de elasticidade na condição de serem equivalentes $\left(\mathrm{E}_{\mathrm{c} 0 \_} \mathrm{A} \equiv \mathrm{E}_{\mathrm{c} 0 \_} \mathrm{B}\right)$, transformada pela equação 2 fica igual

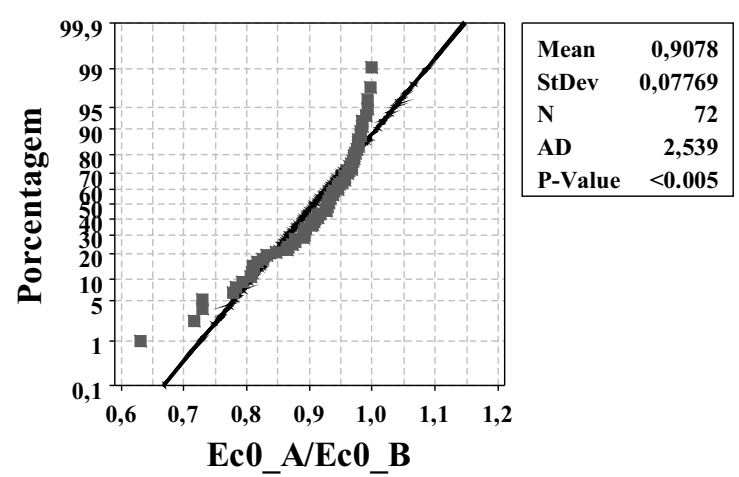

Figura 2 - Resultado do teste de normalidade da razão entre os módulos de elasticidade.

Figure 2 - Results of normality test of the ratio between elasticitymodulus. a 0,1308 , sendo esse o valor utilizado na formulação do teste de hipótese. Os resultados desse teste para verificação da representatividade da unidade $(0,1308$ - valor transformado) sobre o conjunto com os 72 valores da razão entre os módulos de elasticidade indicam o valor do intervalo de confiança igual a $-1,1274 \leq \mathrm{m}$ $\leq-0,9221$ com P-valor igual a 0,000 . O teste de hipótese foi avaliado para um nível de significância de 5\%, utilizando-se a média igual a 0,1308 como hipótese nula $\left(\mathrm{H}_{0}: \mathrm{m}=0,1308\right)$ e a média diferente de 0,1308 como hipótese alternativa $\left(\mathrm{H}_{1}: \mathrm{m} \neq 0,1308\right)$. Pelo fato de o $\mathrm{P}$-valor encontrado ser inferior ao nível de significância $(0,05)$, rejeita-se a hipótese nula, implicando ser significativa a escolha da posição dos relógios comparadores na determinação do módulo de elasticidade na compressão paralela.

O coeficiente $\alpha$ obtido do método dos mínimos quadrados $($ Razão $=\gamma \cdot$ unidade $)$ foi igual a 0,91 , implicando possibilidade de obter um módulo de elasticidade até $9 \%$ diferente ao obtido com o uso de apenas dois relógios comparadores, instalados em duas das quatro faces do corpo de prova.

\section{Transformada de Johnson para Ec0_A/Ec0_B}

Gráfico de probabilidade dos dados originais Selecione uma transformação
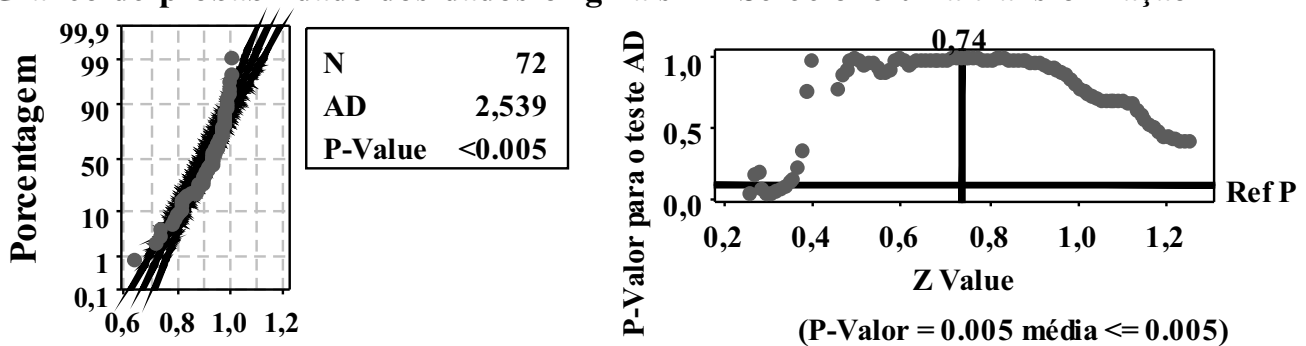

Gráfico de probabilidade dos dados transformados

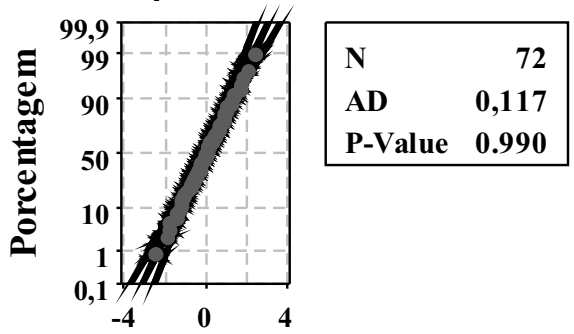

P-Value for Best Fit: 0,989955

Z for Best Fit: 0,74

Best Transformation Type: SB

Figura 3 - Transformada de Johnson da razão entre os módulos de elasticidade.

Figure 3 - Johnson transform of ratio between elasticitymodulus. 


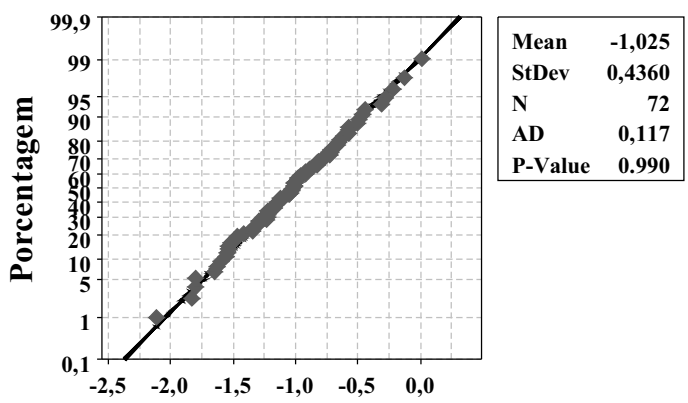

Ec0_A/Ec0_B (dados transformados)

Figura 4 - Resultado do teste de normalidade da razão entre os módulos de elasticidade: dados transformados.

Figure 4-Normality test results for ratio between elasticitymodulus: transformed data.

\section{DISCUSSÕES}

Os resultados deste estudo estão coerentes com os de Ferro et al. (2013), que avaliaram a influência das posições dos relógios comparadores na determinação da rigidez na compressão paralela às fibras para as espécies cambará-rosa (Erisma sp.) e jatobá (Hymenaea stilbocarpa). Os resultados indicaram ser significativa a escolha da posição dos relógios comparadores na determinação do MOE, apresentando uma diferença de $8 \%$ no MOE entre os maiores e menores valores obtidos. Também foram comparados com os de estudos que avaliaram a variação das propriedades mecânicas no interior de uma árvore.

Cruz et al. (2003), para corpos de prova orientados segundo os planos anatômicos da madeira, de híbridos do gênero Eucalyptus, encontraram valores médios de módulo de elasticidade na compressão paralela às fibras que variaram de $20,13 \%$ na direção radial, em que os corpos de prova foram retirados de posições distintas ao longo da seção transversal da árvore, ambos com a direção das fibras na direção paralela. $\mathrm{O}$ incremento nos valores do módulo de elasticidade no sentido da medula para a casca da árvore também foi observado nos estudos de Leonello et al. (2012), Lima e Garcia (2010) e Pimentel et al. (2008).

Com relação à influência de lenhos de reação nas propriedades mecânicas da madeira, podemos destacar o estudo de Ferreira et al. (2006), que encontraram para dois clones de Eucalyptus 6\% de variação entre os módulos de elasticidade do lenho de tração e da madeira normal.

\section{CONCLUSÕES}

Os resultados indicam ser significativa a escolha das faces do corpo de prova na determinação do módulo de elasticidade na compressão paralela às fibras, quando essa for procedida segundo recomendações análogas às da Norma NBR 7190:1997.

Portanto, para que a variabilidade natural da madeira presente no volume do corpo de prova seja representada em apenas um ensaio de compressão paralela às fibras, os valores do módulo de elasticidade devem ser corrigidos (minorados) em $9 \%$ ou realizadas medidas de deformação nas quatro faces do corpo de prova, bem como utilizar, devido às questões de segurança, a menor entre as duas médias obtidas.

\section{AGRADECIMENTOS}

Ao Laboratório de Madeiras e de Estruturas de Madeira (LaMEM) do Departamento de Estruturas (SET) da Escola de Engenharia de São Carlos (EESC) da Universidade de São Paulo (USP), pelos recursos oferecidos para a realização dos ensaios; e à Coordenação de Aperfeiçoamento de Pessoal de Nível Superior (CAPES) e ao Conselho Nacional de Desenvolvimento Científico e Tecnológico (CNPq), pelo apoio financeiro.

\section{REFERÊNCIAS}

ARAÚJO, H.J.B. Relações funcionais entre propriedades físicas e mecânicas de madeiras tropicais brasileiras. Floresta, v.37, n.3, p.399416, 2007.

ASSOCIAÇÃO BRASILEIRA DE NORMAS TÉCNICAS - ABNT. NBR 7190: Projetos de estruturas de madeiras. Rio de Janeiro: 1997.

BALLARIN, A.W.; PALMA, H.A.L. Propriedades de resistência e rigidez da madeira juvenil e adulta de Pinus taeda L. Revista Árvore, v.27, n.3, p.370-380, 2003.

CALIL JUNIOR, C.; LAHR, F.A.R.; DIAS, A.A. Dimensionamento de elementos estruturais de madeira. Barueri: Manole, 2003. 152p. 
CHRISTOFORO, A.L.; ROCCO, F.A.L.; MORALES, E.A.M.; PANZERA, T.H.; BORGES, P.H. Numerical evaluation of longitudinal modulus of elasticity of Eucalyptus grandis timber beams.

International Journal of Agriculture and Forestry, v.2, n.1, p.166-170, 2012.

CRUZ, C.R.; LIMA, J.T.; MUNIZ, G.I.B. Variações dentro das árvores e entre clones das propriedades físicas e mecânicas da madeira de híbridos de Eucalyptus. Scientia Forestalis, n.64, p.33-47, 2003.

FERREIRA, S.; LIMA, J.T.; TRUGILHO, P.F.; SILVA, J.R.M.; LEITE, F.P.; ALTOÉ, T.F. Comparação entre lenho de tração e lenho oposto em Eucalyptus cultivados em diferentes topografias. In: ENCONTRO BRASILEIRO EM MADEIRA E EM ESTRUTURAS DE MADEIRA EBRAMEM, 10., 2006, São Pedro, São Paulo. Anais ... São Pedro: IBRAMEM, 2006. CD-ROM.

FERRO, S.F.; ICIMOTO, F.H.; ALMEIDA, D.H.; CHRISTOFORO, A.L.; ROCCO LAHR F.A. Influência das posições dos relógios comparadores para determinação da rigidez na compressão paralela às fibras da madeira. In: CONGRESSO BRASILEIRO DE CIÊNCIA E TECNOLOGIA DA MADEIRA (CBCM) E DO SIMPÓSIO DE CIÊNCIA E TECNOLOGIA DO ESTADO DO RJ- SIMADERJ, 3., 2013, Petrópolis, Rio de Janeiro. Anais... Petrópolis: Universidade Federal do Rio de Janeiro, 2013. CD-ROM.

GRYC, V.; VAVRČÍK, H. Variability of spruce (Picea abies [L.] Karst.) compression strength with present reaction wood. Journal of Forest Science, v.55, n.9, p.415-422, 2009.

KRETSCHMANN, D.E. The influence of juvenile wood content on shear parallel, compression, and tension perpendicular to grain strength and mode I fracture toughness of loblolly pine at various ring orientation. Forest Products Journal, v.58, n.7/8, p.89-96, 2008.

LAHR, F.A.R. Considerações a respeito da variabilidade de propriedades de resistência e de elasticidade da madeira. 1990. 161f. Tese (Livre Docência em Engenharia de Estruturas) - Universidade de São Paulo, São Carlos, 1990.
LEONELLO, E.C.; BALLARIN, A.W.; OHTO, J.M.; PALMA, H.A.L.; ESCOBAR, J.F. Classificação estrutural e qualidade da madeira do Clone GT 1 de Hevea brasiliensis Muell. Arg. Floresta e Ambiente, v.19, n.2, p.229-235, 2012.

LIMA, I.L.; GARCIA, J.N. Variação da densidade aparente e resistência à compressão paralela às fibras em função da intensidade de desbaste, adubação e posição radial em Eucalyptus grandis Hill ex-maiden. Revista Árvore, v.34, n.3, p.551-559, 2010.

MONTGOMERY, D.C. Design and analysis of experiments. 6.ed. Cary: John Wiley \& Sons, 2005.

PIMENTEL, M.M.; SELEGATO, P.A.M.; GARCIA, J.N.; LIMA, I.L. Variação de propriedades de madeira da Tectona grandis Linn. F. (Teca) em função do espaçamento e da posição radial da tora. Instituto Florestal, n.36, p.19-24, 2008.

ROCHA, J.S. A segurança de estruturas de madeira determinada a partir da variabilidade da densidade básica e de propriedades mecânicas de madeiras amazônicas. 1994. 160 f. Dissertação (Mestrado em Ciências Florestais) Escola Superior de Agricultura "Luiz de Queiroz", Piracicaba, 1994.

SILVA, D.A.L.; LAHR, F.A.R.; FARIA, O.B.; CHAHUD, E. Influence of wood moisture content on the modulus of elasticity in compression parallel to the grain. Materials Research, v.15, n.2, p.300-304, 2012.

STANZL-TSCHEGGA, S.E.; KEUNECKE, D.; TSCHEGG, E.K. Fracture tolerance of reaction wood (yew and spruce wood in the TR crack propagation system). Journal of the Mechanical Behavior of Bio Medical Materials, v.4, n.5, p.688-698, 2010.

TRUGILHO, P.F.; LIMA, J.T.; MENDES, L.M. Influência da idade nas características físicoquímicas e anatômicas da madeira de Eucalyptus saligna. Cerne, v.2, n.1, p.97-111, 1996.

VIDAURRE, G.; LOMBARDI, L.R.; OLIVEIRA, J.T.S.; ARANTES, M.D.C. Lenho juvenil e adulto e as propriedades da madeira. Floresta e Ambiente, v. 18, n.4, p.471-480, 2011. 